\title{
Jean-Luc Gouin
}

$\mathrm{PhD}$, Philosophe québécois

$(2010,1999)$

\section{"Être et Peut-Être. \\ Penser a(u)près (de) Hegel.”}

\author{
Un document produit en version numérique par Jean-Marie Tremblay, bénévole, \\ professeur de sociologie au Cégep de Chicoutimi \\ Courriel: jean-marie_tremblay@uqac.ca \\ Site web pédagogique : http://www.uqac.ca/jmt-sociologue/ \\ Dans le cadre de: "Les classiques des sciences sociales" \\ Une bibliothèque numérique fondée et dirigée par Jean-Marie Tremblay, \\ professeur de sociologie au Cégep de Chicoutimi \\ Site web: http://classiques.uqac.ca/
}

Une collection développée en collaboration avec la Bibliothèque Paul-Émile-Boulet de l'Université du Québec à Chicoutimi

Site web: http://bibliotheque.uqac.ca/ 


\section{Politique d'utilisation de la bibliothèque des Classiques}

Toute reproduction et rediffusion de nos fichiers est interdite, même avec la mention de leur provenance, sans l'autorisation formelle, écrite, du fondateur des Classiques des sciences sociales, Jean-Marie Tremblay, sociologue.

Les fichiers des Classiques des sciences sociales ne peuvent sans autorisation formelle:

- être hébergés (en fichier ou page web, en totalité ou en partie) sur un serveur autre que celui des Classiques.

- servir de base de travail à un autre fichier modifié ensuite par tout autre moyen (couleur, police, mise en page, extraits, support, etc...),

Les fichiers (.html, .doc, .pdf., .rtf, .jpg, .gif) disponibles sur le site Les Classiques des sciences sociales sont la propriété des Classiques des sciences sociales, un organisme à but non lucratif composé exclusivement de bénévoles.

Ils sont disponibles pour une utilisation intellectuelle et personnelle et, en aucun cas, commerciale. Toute utilisation à des fins commerciales des fichiers sur ce site est strictement interdite et toute rediffusion est également strictement interdite.

L'accès à notre travail est libre et gratuit à tous les utilisateurs. C'est notre mission.

Jean-Marie Tremblay, sociologue

Fondateur et Président-directeur général, LES CLASSIQUES DES SCIENCES SOCIALES. 
Cette édition électronique a été réalisée par Jean-Marie Tremblay, bénévole, professeur de sociologie au Cégep de Chicoutimi à partir de :

Jean-Luc Gouin

“Être et Peut-Être. Penser a(u)près (de) Hegel.”

Un texte extrait du livre de Jean-Luc Gouin, Hegel ou de la Raison intégrale. Montréal: Les Éditions Bellarmin, 1999, 223 pp. Texte revu et corrigé de l’épilogue par l'auteur, en janvier 2010.

[Autorisation formelle accordée par l'auteur le 26 mai 2008 de diffuser ce texte dans Les Classiques des sciences sociales.]

91. Courriel : LePeregrin@yahoo.ca

Police de caractère utilisée :

Pour le texte: Times New Roman, 14 points.

Pour les citations : Times New Roman, 12 points.

Pour les notes de bas de page : Times New Roman, 12 points.

Édition électronique réalisée avec le traitement de textes Microsoft Word 2004 pour Macintosh.

Mise en page sur papier format : LETTRE (US letter), 8.5’’ x 11'’)

Édition numérique réalisée le 6 juillet 2008 et modifiée le 26 janvier 2010 à Chicoutimi, Ville de Saguenay, province de Québec, Canada.

\section{fFait avec}

Macintosh 


\title{
Jean-Luc Gouin
}

\author{
PhD, Philosophe québécois \\ "Être et Peut-Être. \\ Penser a(u)près (de) Hegel.”
}

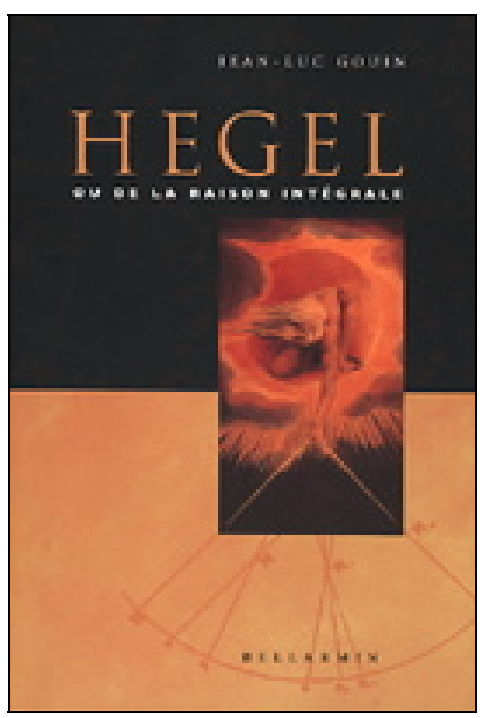

Un texte extrait du livre de Jean-Luc Gouin, Hegel ou de la Raison intégrale. Montréal: Les Éditions Bellarmin, 1999, 223 pp. Texte revu et corrigé de l'épilogue par l'auteur, en janvier 2010. 
Jean-Luc Gouin

$\mathrm{PhD}$, Philosophe québécois

\section{“Être et Peut-Être. Penser a(u)près (de) Hegel.”.}

Un texte extrait du livre de Jean-Luc Gouin, Hegel ou de la Raison intégrale. Montréal: Les Éditions Bellarmin, 1999, 223 pp. Texte revu et corrigé de l'épilogue par l'auteur, en janvier 2010.

Mes propositions sont élucidantes à partir de ce fait que celui qui me comprend les reconnaît à la fin pour des non-sens, si, passant par elles, sur elles, pardessus elles, il est monté pour en sortir. Il faut qu'il surmonte ces propositions. Alors il acquiert une juste vision du monde.

L. Wittgenstein, Le Tractatus logico-philosophicus, prop. 6.54

faute de quoi :

Un livre qui, après avoir tout démoli, ne se démolit pas lui-même, nous aura exaspérés en vain.

Cioran, De l'Inconvénient d'être né

car :

Penser c'est renoncer au savoir.

Schelling

Jacques Derrida estime que «le débat avec Hegel est infini et interminable ». Son collègue Michel Foucault s'interrogeait : "Une philosophie peut-elle encore exister et qui ne soit plus hégélienne ${ }^{1}$ ? »

1 « Hegel est à l'origine de tout ce qui s'est fait de grand en philosophie depuis un siècle - par exemple du marxisme, de Nietzsche, de la phénoménologie et de l'existentialisme allemand, de la psychanalyse. Il inaugure la tentative pour explorer l'irrationnel et l'intégrer à une raison élargie qui reste la tâche de notre siècle. [...] On pourrait dire sans paradoxe que donner une interprétation de Hegel, c'est prendre position sur tous les problèmes philosophiques, politiques et religieux de notre siècle ». Maurice Merleau-Ponty, Sens et nonsens, Paris, Nagel, 1948, p. 109. Quelque vingt ans plus tard, dans un Hegel qui n'a pas vraiment vieilli, François Châtelet rapplique : « Du marxisme au spiritualisme cosmologique en passant par ce qu'on appelle existentialisme 
Or il semble étonnant que ces étoiles de la pensée spéculative des cinquante dernières années demeurassent ainsi médusées par une philosophie que l'on pourrait croire aux antipodes de la leur, imprégnée du « local » et de la « différance ». C'est qu'ils ne sont pas sans savoir que s'opposer à Hegel, c'est tout aussi bien l'accueillir 2.

C'est que, envers et contre tout, le penseur s'est frayé un chemin qui le conduit au fondement, à la source, à la rencontre de tous les pensers. En saisissant la raison à bras-le-corps, par distinction des îlots régionaux d'icelle qui constituent le lot habituel des philosophes $-\mathrm{y}$ compris chez les plus doués et les plus pénétrants, et de tous les temps -, Hegel se voit en effet contraint, en quelque sorte à son corps défendant, à se profiler sur toutes les plages de discours et à s’immiscer dans tous les instruments euristiques de décodage du réel. En réalisant que l' « on ne peut se demander la signification de la pensée, parce que sa signification c'est elle-même, [et qu'] il n'y a rien derrière (Bei dem Gedanken kann nicht nach einer Bedeutung gefragt

[...], aucune des "philosophies" élaborées depuis un siècle et demi n'a pu se déprendre sérieusement des résultats acquis par Hegel [...]. On peut détester le système hégélien, comme Kierkegaard [ou Schopenhauer] ; on ne peut pas aujourd'hui, si l'on pense que l'activité philosophique a un sens, l'éluder. » (Paris, Seuil, Écrivains de toujours, (C) 1968, p. 164). "Aucun avenir ne reniera Hegel », lirons-nous aussi dans le Subjekt-Objekt du respecté Ernst Bloch [1951]. Dès lors, la déclaration d'Alexandre Kojève ne surprendra guère : « L'Histoire ne réfutera jamais l'hégélianisme, mais se contentera de choisir entre ses interprétations opposées. » (cité, p. 125, par René Serreau dans son Hegel et l'hégélianisme, Paris, PUF, 4 e éd., 1972). Cela étant, et afin de bien attester à notre tour de l'ampleur inouïe de cet h-auteur des cimes, nous proposons de marquer cette indiscutable souveraineté intellectuelle du sceau d'un vocable inédit, soit : Hégélémonie. (Eu égard à l'apparat critique et bibliographique, nous renvoyons à l'ouvrage d'où est tiré le présent extrait; lequel en constitue la conclusion formelle telle qu'elle apparaît, hormis léger polissage qui ne modifie rien quant au fond, aux pages 163-169).

2 Dans une entrevue parue dans Le Monde du 31 janvier 1982, J. Derrida lançait avec évidence : "Les critiques frontales se laissent toujours retourner et réapproprier en philosophie. La machine dialectique de Hegel est cette machination même. Elle est ce qu'il y a de plus terrifiant dans la raison. » Maurice Blanchot tenait un propos identique dans son Entretien infini : «Ce besoin de dépassement qui est le cœur de la raison, laquelle est certes dangereuse, terrible, et, à proprement parler, la terreur même. » Paris, Gallimard, 1969, p. 337. 
werden, weil er selbst die Bedeutung ist ; es steckt nichts dahinter) ", il a saisi, du même élan, que l'on ne pourra jamais " s'attaquer » au Logos (substantif grec d'où jaillit à la fois, et d'un seul jet, et le « verbe » - la parole - et la « raison ») que de l'intérieur. C'est-à-dire, par les ressorts exclusifs de celui-ci. Pourquoi ? Parce que la raison, foncièrement, consiste en « la tendance suprême et unique à se trouver et connaître soi-même par soi-même dans tout (das höchster und einziger Trieb, durch sich selbst in allem sich selbst zu finden und zu erkennen) ${ }^{3}$.

Au-delà, à côté et en deça de la pensée, persiste et perdure encore du penser. Nonobstant la forme que cette activité peut, pourrait ou pourra revêtir. Au-delà, au côté ou en deça de la raison, nous baignons encore et toujours dans les eaux érosives du rationnel. Point de repos possible, donc. Le vertige tiendra lieu désormais de religion, pour ainsi dire ontologique, de la condition humaine. Que dis-je ? Chez tout être doué de la faculté d'intelligeance du monde. Aussi le dieu de cette religion confectionnera-t-il son nid nulle part ailleurs que dans le travail de cette "intelligibilisation " même, chevillé au cœur de l'esprit de tout être pensant : "C'est dans la Raison que réside le Divin. Le contenu de la Raison est l'Idée divine, essentiellement le plan de Dieu (In der Vernunft wohnt das Göttliche. Der Inhalt der Vernunft ist die göttliche Idee, hauptsächlich der Plan Gottes) ». En

3 Philo., p. 142 (120), puis Log.-3, p. 371 (552). Également : «L'élément vrai de la pensée n'est pas à chercher dans des symboles arbitrairement choisis, mais seulement dans la pensée elle-même (Das wahrhafte Element des Gedankens ist nicht in willkürlich gewählten Symbolen, sondern nur im Denken selbst zu suchen). », Enc., § 104, add. \#3, p. 541 (222). Dans l'esprit du Séminaire I de Jacques Lacan, l’idée pourrait se décliner comme suit: « Avant la parole, rien n'est, ni n'est pas [...] c'est seulement avec la parole qu'il y a des choses qui sont vraies ou fausses, i.e. qui sont. [...] Il n'y a ni vrai ni faux avant la parole » (Paris, Seuil, 1975 [1954], p. 254). Et c'est ainsi que pour le coup nous retrouvons jusqu'au frisson l'axiome magistral d'Héraclite : « Le maître dont l'oracle est à Delphes ne dit ni ne cache rien, mais seulement signifie. » C'est par le biais de ce vecteur sémiotique que l'on peut du reste comprendre dans toute son acuité la conclusion à la fois impérieuse et envoûtante - et de fait, indépassable - tirée par Hans-Georg Gadamer, depuis son Wahrheit und Methode [1960] : " Le point d'Archimède qui permettrait de soulever de ses gonds la philosophie hégélienne ne pourra jamais être trouvé dans la réflexion ». 
clair : «Rien du penser ne m’est étranger ». Ainsi pourrait se libeller dans un registre qui en outre n'irait pas sans rappeler la manière du Carthaginois Térence («Humani nihil a me alienum puto ») - le fin mot du penser hégélien. Et tout l'art d'exister - de durer dans l'être se jaugera dès lors à l'aune de la capacité à repérer puis maintenir l'équilibre (la Réconciliation, ou Versöhnung) dans la folie constitutive du mouvement perpétuel (ledit « travail ») inhérent à cette intelligibilité : « Le vrai est ainsi le délire bachique dont il n’y a aucun membre qui ne soit ivre; et puisque ce délire résorbe en lui immédiatement chaque moment qui tend à se séparer du tout, - ce délire est aussi bien le repos translucide et simple (Das Wahre ist so der bacchantische Taumer, an dem kein Glied nicht trunken ist, und weil [er] jedes, indem es sich absondert, ebenso unmittelbar auflöst, ist er ebenso die durchsichtige und einfache Ruhe) " ${ }^{4}$.

En d'autres termes, loin de nous germer « dans la tête » par effet de quelque obscure requête "intérieure ", inexpliquée ou présumée inexplicable, voire purement pulsionnelle (« Ça pense plus que ‘je’ ne pense ", écrira plus tard le Husserl des Méditations cartésiennes), nous sommes plutôt dans ce penser qui nous tisse, nous tient, nous contient, nous possède enfin. Et nous 'vertiginise'. Et (penser...) s'en évader, de ce penser, c'est accorder un sens à l'autre-du-penser : c'est amener avec soi ce qu'on croit délaisser. Rien à faire : le pensant est un gladiateur du Sens. Et à aucun titre il ne lui est autorisé de quitter l' " arène » du Logos. Quand bien même il s'efforcerait - comment dire - de penser à autre chose. En bref : on ne se libère point du Sens. Car « le sens de l'expérience humaine se fait à travers nous, mais pas par nous », en déduit promptement Paul Ricœur ${ }^{5}$. Hegel soutiendra pour sa part que :

4 Raison, p. 101 (78), puis Ph.(66), p. 109 et 111, trad. modifiée (108 et 110).

5 In « Hegel aujourd'hui », Études théologiques et religieuses (Montpellier). 1974 (49), p. 353. Une puissante tradition rationaliste se pointillise derrière cette idée, ainsi que nous le rappelle Leibniz dans sa Théodicée (§ 403), publiée dans la langue de Descartes en 1710 : «Nous ne formons pas nos idées parce que nous le voulons; elles se forment en nous [...] suivant notre nature et celle des choses. " « L'homme n'est point à lui-même sa raison et sa lumière, " ajoutait semblablement son contemporain Malebranche dans son Traité de morale. Cf. aussi Philo., p. 75, 102 et 103. 
C'est en agissant de travers que l'homme manifeste le plus sa particularité. Le rationnel est la grand'route où marche chacun et où personne ne se distingue des autres [...]. La plus haute subsistance-par-soi de l'homme consiste à se savoir déterminé sans réserve par l’Idée absolue ${ }^{6}$.

Depuis tout près de deux siècles (La Phänomenologie des Geistes, le premier des grands ouvrages légués par le philosophe à la postérité, fut publiée en 1807), on a tempêté, et souvent avec fureur, contre la raison hégélienne. Mais ne s'est-on pas servi de lui, Hegel, comme d'une égide, voire un alibi inversé, pour tenter désespérément d'éviter de pénétrer la terrible nuit — « cette nuit qu'on découvre lorsqu'on regarde un homme dans les yeux (diese Nacht erblickt man wenn man dem Menschen ins Auge blickt), " disait-il lui-même à Iéna — où séjourne le négatif de cette raison qui n'est au fond que fortuitement hégélienne ? 7

6 «Der Mensch etwas Verkehrtes tut, läßt seine Partikularität am meisten hervortreten. Das Vernünftige ist die Landstraße, wo jeder geht, wo niemand sich auszeichnet [...] Die höchste Selbständigkeit des Menschen, sich als schlechthin bestimmt durch die absolute Idee zu wissen [...] et nous sommes libres lorsque nous le reconnaissons [le rationnel] comme loi et que nous lui obéissons comme à la substance de notre être (und frei sind wir, indem wir es als Gesetz anerkennen und ihn als der Substanz unseres eigenen Wesens folgen). » Droit, § 15, add., p. 82 (67), Enc. § 159, add., p. 589 (304) et Raison, p. 140 (115). À rapprocher certainement encore de ce fragment d'Héraclite, que notre auteur estimait d'ailleurs beaucoup : «Aussi faut-il suivre le logos commun. Mais bien qu'il appartienne à tous, le vulgaire n'en vit pas moins comme si chacun avait une intelligence particulière. » Traduit par Jean Voilquin, in Les penseurs grecs avant Socrate. Paris, GarnierFlammarion, 1964, p. 74. Voir Enc., § 24, add. \#2, p. 477, Droit, § 317, p. 318, et même Philo.-7, p. 2117. Hegel avait également écrit - nous citons de mémoire son Histoire de la Philosophie - que l'originalité consiste à produire quelque chose de tout à fait universel. Bref, le général comme le Grand général - le cinq étoiles, si je puis dire - de l’Être.

7 La Philosophie de l'Esprit - Realphilosophie [1805-1806]. Traduction et notes de Guy Planty-Bonjour. Paris, PUF, Épiméthée, 1982, p. 13 (Gesammelte Werke, Band 8, p. 187). Merleau-Ponty, dans l'article déjà cité de Sens et Non-Sens, p. 117, s'est visiblement inspiré de ce passage pour avancer une idée non moins terrible qu'elle reste hégélienne : « La seule expérience qui me rapproche d'une conscience authentique de la mort, c'est l'expérience d'autrui, puisque sous son regard je ne suis qu'une chose, comme il n'est qu'un morceau de monde sous mon propre regard. » On pourrait sans 
Une raison cachée, mystérieuse ou impénétrable - cette raison analogue à ce Dieu de l'abstraction divine - laisse place à l'imagination, aux "on-verra-plus-tard”, à la sécurité $d u$ nonsavoir. Car, d'une part, par sa nature propre de raison elle incarne le sens, et par là permet des jours heureux d'insouciance en apaisant mes angoisses d'être-de-finitude («Si Dieu existe, tout est bien... », écrit Marc-Aurèle dans ses Pensées, IX, 28, 3). D'autre part, son caractère inaccessible m'autorise à "laisser aller », à faire confiance — et m'évite surtout d'en faire la rencontre qui risquerait de me faire mourir de ne pas mourir, pour paraphraser Thérèse d'Avila ${ }^{8}$. Ainsi, et de la sorte :

Nous plaçons Dieu au-delà de notre conscience rationnelle, nous nous trouvons du même coup affranchis du souci de connaître sa nature et de reconnaître la présence de la Raison dans l'histoire. La voie est ainsi libre au jeu des hypothèses arbitraires: la pieuse humilité sait fort bien ce qu'elle gagne à de tels renoncements ${ }^{9}$.

doute à ce sujet prendre plaisir à se remémorer l'histoire du "trou de serrure" de Jean-Paul Sartre.

8 Expression reconquise plus tard par Paul Éluard, qui en fit le titre d'un recueil de poèmes. Au reste, n'est-ce pas cette vertigineuse angoisse que décrit Dostoïevski dans ses Possédés lorsque, dans les dernières pages, il fait dire à Stavroguine : «Si j'atteignais à cette liberté, j'étais perdu » ? Le Nietzsche de Ecce homo [1888] a trouvé une formule foudroyante pour exprimer le sentiment d'une pareille expérience des limites : «Ce n'est pas le doute, c'est la certitude qui rend fou [...] Nous avons tous peur de la vérité. » La Philosophie du marteau, avez-vous dit ? Gallimard, NRF, p. 47.

9 "Wird Gott jenseits unseres vernünftigen Bewußtseins gestellt, so sind wir davon befreit, sowohl uns um seine Natur zu bekümmern, als Vernunft in der Weltgeschichte zu finden; freie Hypothesen haben dann ihren Spielraum. Die fromme Demut weiß wohl, was sie durch ihr Verzichten gewinnt. » Raison, p. 60 (41), nous soulignons. Et en récidive : «Au lieu de soumettre sa pensée au travail de l'étude, de plier sa volonté à une discipline pour la transformer en une obéissance librement consentie, il est plus facile de renoncer à la connaissance de la vérité objective, de conserver un sentiment d'oppression et, par là, de sauver son amour-propre (Statt sein Meinen mit der Arbeit des Studiums zu bezwingen und sein Wollen der Zucht zu unterwerfen und es dadurch zum freien Gehorsam zu erheben, ist es das Wohlfeilste, auf die Erkenntnis objektiver Wahrheit Verzicht zu tun, ein Gefühl der Gedrücktheit und damit den Eigendünkel zu bewahren). » Droit, § 270, rem., p. 273 (419420). Or, de rétorquer finement sur ces matières le Cocteau du Rappel à 
En définitive, je ménage la chèvre du Sens et le chou de la Tranquillité d'esprit : Je pense récupérer du sens dans la dispense de penser. Et c'est alors « la théologie [qui] accorde ce que la spéculation refuse [...] Celle-là est en effet la fuite de celle-ci. » Il n'y a que de mauvaise foi 10 .

Par ailleurs, le tragique constitutif de la raison authentique (manifeste, transparente à elle-même), on l'aura déjà subodoré, c'est qu'elle n'offre pas d'issue plus que n'en laisse le non-sens ou même l'absurde. Sens ou non-sens, tous deux me chavirent et se jouent de moi dans un univers où je n'a[i] choisi ni l'un, ni l'autre. Non, le sens n'est pas moins noueux que le non-sens : je suis dans le sens comme

l'ordre, « le mystère est une position trop favorable pour qu'un esprit bien élevé s’y maintienne ».

« On appelle profond un puits dont on ne distingue pas le fond, c'est ainsi que ce qui est caché paraît profond aux hommes; il y a là-dessous de la profondeur, pense-t-on ; il se peut aussi qu'il n'y ait rien derrière ce qui est caché [ou, plus précisément : derrière ce qui occulte] (Man einen Brunnen tief nennt, dessen Boden man nicht sehen kann -, so kommt leicht den Menschen das tief vor, was verborgen ist; dahinter stecke Tiefes. Wenn es versteckt ist, so ist auch der Fall möglich, daß nichts dahinter ist). » Philo., p. 241 (211). "Ce qui est caché n'est jamais que ce qui manque à sa place » estimait Lacan (Écrits, Paris, Seuil, 1966, p. 25) avec le doigté qu'on lui connaît. On perçoit, si ce n'était fait, combien il est aisé d'abonder dans le sens des Bruno Bauer et des Michel Bakounine, lequel tenait Hegel, dans Étatisme et Anarchie [1873], comme «le véritable père de l'athéisme scientifique moderne.» La vision théogonique façon Émile Bréhier n'est pourtant pas ridicule. Ainsi va l’hégélianisme.

10 "Theologie gewährt, was die Speculation versagt [...] Denn jene ist die Flucht ausdieser. » Notes et fragments, p. 69 (68) et Ph.-2, p. 85 (392). «Il y a une consolation religieuse, il n’y a pas de pensée religieuse, » ajouterait de Gaulle en contrepoint. Il faut admettre en effet avec Anne Hébert, grande dame de la littérature, que « nous craignons, d'une terreur égale, la lumière en nous qui force la pensée, la suscite et lui donne forme, et le passage au grand jour de cette pensée devenue expression et langage ». Tâche immense, on le sait : " La vérité pensée a comme telle à se prouver à la pensée (Die gedachte Wahrheit als solche sich dem Denken zu bewähren hat). » Enc., § 159, add., p. 589-590 (306). 
l'oiseau en cage 11 ! Sens et non-sens s'unissent dans un même sansfond (Ungrund) qui, icelui, me pétrit de part en part sans qu'il soit possible d'arpenter d'autres avenues. Le sens est dictatorial par essence 12 .

11 «Souvent Hegel me semble l'évidence, mais l'évidence est lourde à supporter », nous confie Georges Bataille dans Le Coupable. Derrida sait bien pourquoi, et il le dit dans une économie de mots remarquable: "La révolution contre la raison ne peut se faire qu'en elle». L'Écriture et la différence, Seuil, 1967, p. 59. Bernard Bourgeois, quoique sous un phrasé à la sémantique fuyante, confirme l'inéluctable dans la présentation à sa traduction de l'Encyclopédie: "L'objection qui demande ses raisons à l'affirmation de la vérité absolue du concept de la Logique se situe d'elle même en dernière analyse à l'intérieur de la conscience rationnelle ou pensante. » Enc., p. 104. Dans les termes lacaniens de "L'instance de la lettre », cela signifie " qu'il n'est aucune signification qui se soutienne sinon du renvoi à une autre signification ». On revient au principium reddendae rationis de Leibniz. Et que Hegel traduira comme suit : « Expliquer et concevoir signifie d'après cela montrer quelque chose comme médiatisé par quelque chose d'autre (Erklären und Begreifen heißt hiernach, etwas als vermittelt durch ein Anderes aufzeigen). » Enc., § 62, rem., p. 325 (149). Et c'est là aussi - dans l'ombre de Schelling, et dans un trait fulgurant faut-il le rappeler: "Penser c'est renoncer... » - tout l'objet de notre livre. Qui aura tenté de préserver équilibre - la sérénité dans les délires de Bacchus - en s'aventurant sur peau de banane au détour de chaque phrase.

12 «Peut-être ne savais-tu pas que je suis logicien? », confessera, sourire sardonique à la commissure des lèvres, le diable de La divine Comédie (Inferno, chant XXVII). De fait, et comme le remarquait Jacques Rolland de Renéville, par son in-sondabilité même le sans-fond abyssal (grundlos) se montre également frivole parce que sans fondement : "Le fond se dérobe au sondage et par là il fait défaut. » Aventure de l'Absolu, La Haye, Martinus Nijhoff, 1972, p. 315. Le professeur Ladrière, de Louvain, aurait avancé la jolie façon de dire, à savoir qu'il est "fondant". Le fond-sans-fond (der Ungrund) se fond au fond sans-fondement. Derrière le sérieux du philosophe, l'éclat de rire. Rabelaisien! Mais au fait : le rire de Qui ? Et enfin, de Quoi ?

C'est ici sans doute que s'insèrerait le jeu de Heidegger, qui est « l'essence même de l'être [...or] le Jeu du monde (das Welten von Welt) ne peut être, ni expliqué par quelque chose d'autre, ni appréhendé dans son fond à partir de quelque chose d'autre [...] causes, fondements et choses de ce genre demeurent inadéquats au Jeu du monde [...car] le "parce que" disparaît dans le Jeu. » Questions I, Essais et Conférences et Le Principe de Raison (voir bibliographie de l'ouvrage), pages 300, 214 et 243 respectivement. En d'autres termes : « Le sens est en fonction du jeu, il est inscrit en un lieu dans la configuration d'un jeu qui n'a pas de sens » (Jacques Derrida derechef, qui 
Alors, devant le non-sens : à quoi bon exister ? Et devant le sens, ai-je autre choix que celui de devenir « collabo » - autre alternative à la réconciliation, croix au cœur ou rose dans la croix ${ }^{13}$ ?

pour l'occasion cite Bataille, op. cit.,p. 382). D’où incidemment (ne nous arrêtons pas en si bon chemin), chez Michel Serres, la transposition conséquente de ce constat théorique sur le plan pratique (la nano-science rencontrant en quelque sorte la Megapolis) ; lequel en effet reconnaît, et non sans discernement, que l'ordre n'est en définitive rien d'autre... qu' « un cas particulier du désordre ». Tout cet univers de sens dans la tempête - ce fond évanescent et tout léger, ce Jeu tournoyant le plus sérieusement du monde autour de lui-même, ce désordre bien cordé -, Hegel en aura déjà battu le rappel pour l'inscrire à jamais, on le sait maintenant, dans la représentation saisissante et indélébile de l'allégorie du vrai considéré comme "délire bachique » (bacchantische Taumer). Nulle licence ou folie, ni outrance ou excès, par conséquent, n’échappent à la raison. Qui garde le dernier mot pour elle. L'égaré ne s'étourdit, ne trébuche ou ne se perd que dans ce petit carré de sable déjà nommé « arène de sens ». Fût-ce l'infini même. À l'image du pays du poète Gilles Vigneault, qui « ne tient pas plus de place qu'un brin d'herbe sous l'hiver ».

(En écharpe, il faudra signaler que l'on n'a pas toujours su faire la nuance chez les commentateurs entre ce que nous appelions à l'instant la dictature du sens d'une part, la personnalité propre du philosophe - que d'aucuns ont qualifié de « secrétaire de l'Absolu » - d'autre part. Un “dicteur”, sans doute. Aller plus avant dans la paronymie devient cependant déshonnête. À moins que l'on ne songeât à forger “didacteur”, depuis didaktikos - didactique / enseignement.)

13 « Reconnaître la raison comme la rose dans la croix du présent et se réjouir d'elle, c'est là la vision rationnelle qui constitue la réconciliation avec la réalité (Die Vernunft als die Rose im Kreuze der Gegenwart zu erkennen und damit dieser sich zu erfreuen, diese vernünftige Einsicht ist die Versöhnung mit der Wirklichkeit), » écrit le philosophe en s'inspirant explicitement de la symbolique des rose-croix. Préface à Droit, p. 57-58 (26-27). On rejoint le sage d'Alexandre Kojève ou le fou dont parle Friedrich Nietzsche, et qui paya cher son audace à affronter la vérité les mains nues. Il y a ici quelque chose comme un burn-out du sens. Et certes, nous dit Hegel dans un dernier aphorisme aux accents jacobiens, «l'homme voudrait savoir pourtant pourquoi la nature en est restée avec lui à mi-chemin et ne lui permet que de pressentir là où il exige certitude (Der Mensch möchte er doch wissen, warum die Natur mit ihm auf halbem Wege stehen geblieben und ihn da nur ahnen läßt, wo er Gewißheit fordert). " Notes et fragments, p. 71 (70). D'où le désespoir du chercheur d'intelligibilité. Nous savons en effet désormais, avec Kojève, que si « le Philosophe est mécontent parce qu'il ne sait pas ce qu'il 
Comment sortir enfin vainqueur de la défaite Comment réconcilier sans pourtant renoncer Comment affronter lumière des ténèbres

N'y a-t-il de Liberté que d'obéissance... ? 14

$$
* * *
$$

\section{Quelques textes à la fois de même assonance et du même auteur}

- Le Commissaire et le Détective (compte-rendu de deux biographies sur Hegel En guise d'intromission à l'univers hégélien)

- «Der Instinkt der Vernünftigkeit. De l'inaliénabilité de la rationalité » (à la fois une porte d'entrée à Hegel et une introduction à la rationalité philosophique de manière générale)

- «Radicalité du sens et altérité en tous sens. Entropie et philentropie chez Georg W.F. HEGEL » (incursion au coeur du penser hégélien)

- «Die Dialektik des Staates. Hegel ou de la Liberté constitutive de la Raison » (les impératifs politiques de la rationalité telle que comprise par Hegel - à venir en 2011)

- «Aimer Penser Mourir : Hegel, Nietzsche, Freud en miroirs » (extrait)

- « Hegel en débat - Monodialogue Michel Onfray / J.-L. Gouin » (un quasi échange épistolaire portant sur le philosophe Hegel)

- Le « Rond de Science » (variations sur la notion d’Encyclopédie)

- De la malhonnêteté intellectuelle (mot d'humeur de rigueur)

- Recensions / analyses critiques (essais / littérature)

- Bibliographie introductive à l’œuvre hégélienne (Hegel sans coups ni blessures...)

\section{Fin du texte}

veut, » comme on le croit parfois, c'est bien « parce qu'il ne sait pas ce qu'il veut ». Introduction à la lecture de Hegel (Gallimard, 1947), p. 280.

- Nous sommes tous des Prométhée enchaînés •

14 «C’était écrit, mais nous avons signé ». William Shakespeare, César. 